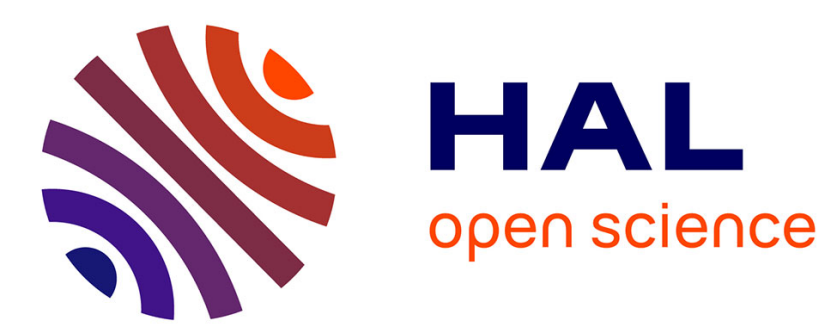

\title{
A micro-structural model for dissipation phenomena in the concrete
}

\author{
Daria Scerrato, Ivan Giorgio, Alessandro Della Corte, Angela Madeo, Ali \\ Limam
}

\section{- To cite this version:}

Daria Scerrato, Ivan Giorgio, Alessandro Della Corte, Angela Madeo, Ali Limam. A micro-structural model for dissipation phenomena in the concrete. International Journal for Numerical and Analytical Methods in Geomechanics, 2015, 39 (18), pp.2037-2052. 10.1002/nag.2394 hal-01194317

\section{HAL Id: hal-01194317 https://hal.science/hal-01194317}

Submitted on 5 Sep 2015

HAL is a multi-disciplinary open access archive for the deposit and dissemination of scientific research documents, whether they are published or not. The documents may come from teaching and research institutions in France or abroad, or from public or private research centers.
L'archive ouverte pluridisciplinaire HAL, est destinée au dépôt et à la diffusion de documents scientifiques de niveau recherche, publiés ou non, émanant des établissements d'enseignement et de recherche français ou étrangers, des laboratoires publics ou privés. 


\title{
A micro-structural model for dissipation phenomena in the concrete
}

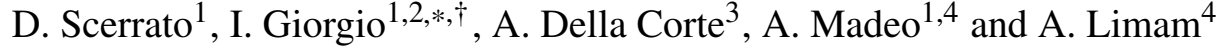 \\ ${ }^{1}$ International Research Center on Mathematics and Mechanics of Complex Systems, Università dell'Aquila, \\ Cisterna di Latina, Italy \\ ${ }^{2}$ Department of Structural and Geotechnical Engineering, Università di Roma La Sapienza, 18 Via Eudossiana, \\ Rome, Italy \\ ${ }^{3}$ Department of Mechanical and Aerospace Engineering, Università di Roma La Sapienza, 18 Via Eudossiana, \\ Rome, Italy \\ ${ }^{4}$ Université de Lyon-INSA, 20 Av. Albert Einstein, Villeurbanne cedex, France
}

\begin{abstract}
SUMMARY
In the present paper, a constitutive model for the description of the dissipation in the concrete is provided. The theoretical description is based on a micromorphic model in which the microstructure is constituted by a kinematical scalar descriptor $\phi$ whose time derivative is linked to a dissipative potential. The scalar $\phi$ can be interpreted as the relative displacement between two opposite faces of the microcracks, and our physical interpretation of dissipation is indeed linked to the friction force (in a mixed Coulomb-type and viscous-type behavior) between them. To evaluate the effects of bending on the dissipation, the 3D model is then reduced by means of standard Saint-Venant's procedure in case of combined compression and bending over a cylindrical domain. A qualitative analysis of the reduced ODEs model is then provided. Numerical results showing comparison between different types of dissipative force and between pure compression and combined compression and bending are included in a dedicated section. Finally, the proposed model and our physical interpretation of the dissipation are supported by some experimental data concerning standard concrete and a concrete enriched by adding to the mixture a filler constituted by micro-particles capable of improving the dissipative behavior of the material. Measured data show very good fit with our theoretical previsions and provide a sufficiently sound basis for further deepening of the theoretical description of the considered phenomena.
\end{abstract}

KEY WORDS: concrete; dissipation of energy; micromorphic continua; internal friction; cyclic load

\section{INTRODUCTION}

Dissipative phenomena still pose a significant amount of scientifically open questions. As usually is the case, open questions naturally entail the possibility to investigate new promising applications - in otherwise hardly conceivable directions - as soon as a reasonable amount of reliable evidence is at disposal and a suitable theoretical framework is developed. In particular, the possibility to reach an optimized dissipation from mechanical to internal energy to achieve damping effects in building materials, and particular in concrete, is of course a very 'hot' scientific topic (an important general reference on concrete is [1]; for a second gradient approach for damage in concrete, see $[2,3])$. The kind of dissipation investigated in the present paper is related to internal friction, and potentially has a relevant role in Civil Engineering, and in particular in cases of large structures,

*Correspondence to: I. Giorgio, Department of Structural and Geotechnical Engineering, Università di Roma La Sapienza, 18 Via Eudossiana, Rome, Italy.

${ }^{\dagger}$ E-mail: ivan.giorgio@uniroma1.it 
when a considerable amount of concrete is employed. The external actions for which the considered dissipation could be important include the case of seismic solicitation. In this case, when an overall motion is imposed to the structure, a distributed dissipation due to the characteristic of the material is indeed advantageous over the employment of localized dampers.

Of course, internal friction plays a relevant role in many different fields, for instance concerning porous elastic solids containing a viscous fluid $[4,5]$ where internal friction changes, even in some cases heavily, the mechanical behaviour of the media.

From an historical perspective, concretes have been usually considered as elasto-damageable materials [6] or elasto-plastic media [7], while more recently, some coupling between damage and plasticity has been taken into account [8-11]. With the same perspective but in a different context, the evolution of the damage can be treated and modelled as a phase transition (see e.g. [12]). These purely phenomenological models have been also recently revisited to introduce more physically based arguments by taking into account the underlying microstructure through some homogenization techniques $[13,14]$. Besides internal friction, therefore, other dissipation phenomena have been taken into account in the literature, such as concrete damage, friction of macrocrack faces associated to permanent strain (plasticity) and plasticity of rebars. Among these possibilities, the kind of internal friction herein considered occupies a special place, as it is not linked to irreversible changes. Damage-associated and permanent-strain associated dissipation can be seen as the 'emergency brake' of a structure under heavy solicitation, capable to avoid overall collapsing. Internal friction because of microcrack faces' sliding represent instead a prophylactic element, which can be able to delay the time at which the emergency brake must work, potentially preventing the costs associated to repair work.

The problem of the cyclic behaviour is still an open question. More precisely, some experiments are showing that, even inside the so-called elastic domain (i.e. the stress domain delimited by the yield surface), stress-strain cycles can be slightly open, so exhibiting some internal dissipation (on general characteristics of dissipation in solid materials the reader is referred to [15-18], and references therein). This feature might be viewed as a paradox of limited importance; but, for the sustainability of constructions under seismic events, this small dissipation appearing for cycles inside the yield surface is an important aspect. Indeed, the use of a concrete able to dissipate as much energy as possible for a small strain level will have in practice a considerable impact. Moreover, these considerations also apply in case of other environmental solicitations which can determine similar effects (as those considered, e.g. [19]).

The aim of the present work is to link this abstract possibility to three sets of clear-cut scientific ideas, which are as follows:

1. a (new) microstructured constitutive model for an enriched concrete, in the framework of which dissipation/damping can be naturally considered and do not involve any significant effect on the strength of the material in the long run;

2. a numerical model providing realistic and computationally stable simulations of the behaviour of the system in practically relevant conditions;

3. a set of experimental data sound enough to support the previously mentioned theoretical and numerical results.

The idea of employing a micromorphic model to theoretically 'capture' the nature of energy dissipation is, in our view, a very natural one (the literature on micromorphic materials is by now a very large chapter in continuum mechanics; as a baseline, the reader can see [20-25]). Indeed, if one is interested in a material capable to dissipate energy without losing strength, one can reasonably start from a formal tool in which dissipation is described by the behaviour of variables not directly related with mechanical characterization (for the rational formulation of the yield criteria of the concrete, it is in our opinion very useful the method developed in [26]). In this connection, because strength is described by a set of well-defined bulk properties, it is quite natural to think to link the dissipation to lesser scale ('micro') phenomena, whose coupling with the bulk properties determines dissipation, entails damping and does not change 'macro' properties. 
In our experimental work, we studied the internal dissipation in concrete under cyclic loading inside the yield surface. According to the experiments [27, 28], in which large campaigns of triaxial tests on enriched concrete were performed, the addition of specific granular fillers can significantly increase the dissipation, opening a way to improve the behaviour of concrete in relation with seismic loading. As for our theoretical approach, we considered the 'macro' structure (i.e. the concrete skeleton) as an isotropic linear elastic medium which includes some microcracks whose opposite faces are able to dissipate energy by mixed Coulomb-type and viscous friction during their sliding under cyclic loading. The dimension of the employed micro-filler is of the order $10^{0}-10^{1} \mu \mathrm{m}$, while the observed length of the micro-cracks ranges between 1 and $500 \mu \mathrm{m}$ [27]. We also recall that the microcracks here considered have to be seen as pores naturally present (for chemical reasons) in the concrete, rather than as true cracks arisen as a consequence of micro-fractures occurred after the drying of the concrete. This is consistent with the experimental evidence showing that they already exist when the drying of the concrete has just completed [1]. It is then natural to assume that some micro-particles can be found inside the microcracks in a non-negligible amount if their percentage is high enough, and therefore to imagine that the micro-particles are capable to modify the aforementioned considered frictional phenomena, enhancing it if their amount and geometrical characteristics are suitable and worsening it if they are too much so as to hinder the relative motion of the faces of the micro-cracks.

To characterize the micro-slide of the microcrack faces, a new scalar variable $\phi$ is introduced. Of course this new variable is playing a basic role in the model, which justifies the fact that it has its own specific constitutive equation. This parameter can be interpreted as an internal variable (as conceived in similar models, see e.g. [29, 30]) or as a true micro-structural parameter (see e.g. [20, 23, 31-34]). The first interpretation implies that the deformation energy only depends on the variable $\phi$, while in the second case, also a dependence on the spacial derivative $\nabla \phi$ can be taken into consideration to account for longer range effects due to the micro-deformation in the neighbourhood of a considered representative elementary volume (REV). Related problems concerning the high heterogeneity characterizing the concrete may be linked to the high variability of the mechanical properties of this material at the micro-scale level, and can thus be modelled by introducing in the strain energy higher-order derivatives of the displacement field (see e.g. [34-42]). Indeed, various higher-gradient and micromorphic models have been proposed in the last decades to investigate different effects of physical systems with microstructure (see e.g. [43-47]).

The newly introduced variable $\phi$ is also included in the expression of the local Coulomb friction force to obtain a full coupling between the skeleton and the microcracks (as general references on internal friction the reader can refer to [48-52]). Eventually, a comparison between theoretical modelling, numerical analysis and experimental results will be provided; the latter, in particular, to exhibit the influence of the addition of a suitable filler on the dissipative behaviour of enriched concrete. Some prospects on future developments to enlarge the frequency range of modelling validity will also be given in the conclusions.

\section{A MODEL OF INTERNAL FRICTION FOR THE CONCRETE}

\subsection{The model}

Concrete is a composite material, basically constituted by a (variable) mixture of aggregate, cement and water. Its physical properties are of course determined by the proportion between the aforementioned elements and by the possible presence of other additives. A variety of mathematical approaches have been developed in order to describe concrete. The characteristics of the employed model are varying depending on different features like, for example, the type of loading, the available experimental results to calibrate material parameters and computational feasibility. Actually, as often is the case when considering theoretical models from hard sciences, the choice of the formal tools employed is also dependent on the questions to answer which one is constructing the model. As already said, in the present paper, the focus is mostly directed on the description of dissipation phenomena. A few models, mostly based on fracture/dislocation phenomena, have been proposed in literature to describe dissipation in concrete (see e.g. [53-58]). 
In our physical interpretation, the dissipation is in fact conceived as due to internal friction between the overlying faces of microcracks distributed in a uniform and isotropic way in the concrete. Moreover, the average characteristics of the microcracks are assumed to not change in time (see on this respect $[59,60]$ ). These assumptions are reasonably verified in the bulk of the material, far enough from the boundary. We remark that the size of the structures for which the presented model is thought is such that the boundary effects can be neglected when considering a variable that is approximately proportional to the total volume of material, as the amount of dissipated energy due to internal friction is. These assumptions, as already observed in Scerrato et al. [61], allow to suitably describe the dissipation through the introduction of a single scalar kinematic micro-descriptor $\phi$, which can be interpreted as the average relative displacement of the overlying faces of a microcrack (for a somewhat similar approach, see [62,63] and [64]). In the proposed model, the energy is thus characterized by two variables: the ordinary ('macro') strain tensor $\boldsymbol{E}$ and the new ('micro') scalar variable $\phi$ :

$$
\Psi=\frac{1}{2} \lambda[\operatorname{tr}(\boldsymbol{E})]^{2}+\mu \operatorname{tr}\left(\boldsymbol{E}^{2}\right)+\frac{1}{2} k_{1} \phi^{2}+\frac{1}{3} k_{2} \phi^{3}+\frac{1}{4} k_{3} \phi^{4}+\alpha \phi \sqrt{I_{2}^{(d)}}
$$

where $\lambda$ and $\mu$ are the Lamé parameters for linearly elastic isotropic materials, $\boldsymbol{E}$ is the linearized Green-Lagrange strain tensor and the scalar $I_{2}{ }^{(d)}$ is the second invariant of the deviatoric strain tensor $I_{2}{ }^{(d)}=\frac{1}{2} \operatorname{tr}(\operatorname{dev} \boldsymbol{E} \operatorname{dev} \boldsymbol{E})$, which can be interpreted as a local shear deformation measure. Some words have to be devoted to the particular form we have chosen for the stored strain energy, especially regarding the polynomial part. If we replace it by a simple quadratic term $1 / 2 k_{1} \phi^{2}$, the resulting elastic force is linear, which is a reasonable assumption in the presence of small deformations in a static regime. In our context, the assumption of small deformations is kept, but the one relative to a static solicitation has to be relaxed, because we are interested in dynamic (cyclic) solicitations. In this case, experimental evidence shows that the relation between stress and strain is not linear even for small deformations [27]. The simpler form to be assumed, which implies a nonlinear behaviour with a well-posed (i.e. convex and positive-definite) energy, is the polynomial one that we selected. As we will see, this form is able to account for the experimental evidence we will provide in a reasonably good way.

The strain energy density for an isotropic material can also be expressed in terms of the bulk modulus $K$ and the shear modulus $\mu$ [65], perhaps in a more convenient way than employing the pair of Lamé's parameters [66]. However, considering that the examples of our numerical applications and experimental tests concern a Saint-Venant cylinder, we preferred, for the sake of simplicity, to employ the usual Lamé's pair. The resulting Lagrangian functional is then

$$
\begin{aligned}
\mathscr{L}=\mathscr{K}-\Psi= & \frac{1}{2} \rho(\dot{\boldsymbol{u}})^{2}+\frac{1}{2} \rho_{\phi}(\dot{\phi})^{2} \\
& -\left(\frac{1}{2} \lambda[\operatorname{tr}(\boldsymbol{E})]^{2}+\mu \operatorname{tr}\left(\boldsymbol{E}^{2}\right)+\frac{1}{2} k_{1} \phi^{2}+\frac{1}{3} k_{2} \phi^{3}+\frac{1}{4} k_{3} \phi^{4}+\alpha \phi \sqrt{I_{2}^{(d)}}\right)
\end{aligned}
$$

In the present paper, a dissipative behaviour is also considered. Indeed, an internal friction force density $\tau$ is introduced, which is a generalization of the Coulomb-type friction model chosen to match experimental observations and predict the dynamic behaviour of concrete. In the Coulomb model, the friction force is proportional to the normal contact force and its direction is always opposite to that of the velocity. In our case, the normal force is the hydrostatic pressure $\sigma_{n}$ (i.e. the compressive stress), while the velocity to be considered is that of the sliding between the opposite faces of the micro-cracks, that is, $\dot{\phi}$. Therefore, $\tau$ can be written as $\tau=\zeta \operatorname{tr}(\boldsymbol{E}) \tanh (\eta \dot{\phi})$, where $\zeta$ and $\eta$ are constitutive constants, and the amplitude of the internal friction is proportional to $\operatorname{tr}(\boldsymbol{E})$. Specifically, we can write $\zeta \operatorname{tr}(\boldsymbol{E})$ as $\varpi \mu_{k} \sigma_{n}=\varpi \mu_{k} K \operatorname{tr}(\boldsymbol{E})$, which is the product of the specific surface $\varpi$ of the microcracks (i.e. the surface area of microcracks per unit volume of concrete), the friction coefficient $\mu_{k}$ and the compressive stress $\sigma_{n}$. The compressive stress, in turn, is proportional to the trace of the strain tensor, the constant of proportionality being the Bulk Modulus $K$. The function tanh, instead of the usual sign function, is introduced not only to smoothen the term but also 
to be able to consider a viscous effect in the low velocity range (for a viscous approach for friction in different contexts, see e.g. [31, 67-69]). The slope $\eta$ can be used to modulate the extension of the viscous range and can be interpreted as the inverse of a reference velocity (for further interesting mathematical considerations about modelling of friction, see e.g. [70, 71]).

\subsection{Reduction procedure for combined compression and bending}

As already observed in [61], one can reduce the model using the Saint-Venant theory for simple compression load on a cylindrical reference configuration. In the present work, we consider a slightly more general context by introducing, besides the compression, a bending load. If one wants to further generalize the results presented below to the case of non-vanishing shear deformations, some non-trivial problem may arise, to address which a number of theoretical tools have been developed (see e.g. [72]).

We consider isochronous motions and arbitrary variations for the generic component $\epsilon$ of the strain tensor and for the variable $\phi$. The principle of least powers $[73,74]$ leads then to the following reduced procedure. We start with a cylindrical domain of height $H$ and radius $R$. By suitably choosing the reference frame, the strain tensor $\boldsymbol{E}$ and the displacement $\boldsymbol{u}$ considered in the Saint-Venant theory can be explicitly expressed by

$$
\boldsymbol{E}=\left(\begin{array}{ccc}
-v\left(\frac{\epsilon_{b}}{R} x_{1}+\epsilon_{c}\right) & 0 & 0 \\
0 & -v\left(\frac{\epsilon_{b}}{R} x_{1}+\epsilon_{c}\right) & 0 \\
0 & 0 & \left(\frac{\epsilon_{b}}{R} x_{1}+\epsilon_{c}\right)
\end{array}\right), \quad \boldsymbol{u}=\left(\begin{array}{c}
-v\left(\frac{\epsilon_{b}}{R} x_{1}+\epsilon_{c}\right) x_{1} \\
-v\left(\frac{\epsilon_{b}}{R} x_{1}+\epsilon_{c}\right) x_{2} \\
\left(\frac{\epsilon_{b}}{R} x_{1}+\epsilon_{c}\right) x_{3}
\end{array}\right),
$$

where $\epsilon_{b}$ and $\epsilon_{c}$ are respectively the bending and compressive strain and $v=\frac{\lambda}{2(\lambda+\mu)}$ is the Poisson coefficient. Given the form of the employed coupling term, our scalar microstructural variable $\phi$ is also linear in $x_{1}$ and thus of the form $\phi=\left(\phi_{b} \frac{x_{1}}{R}+\phi_{c}\right)$. Substituting expressions (3) in Equation (2), the principle of least powers implies the following reduced form of the model:

$$
\left\{\begin{array}{l}
M_{c c} \ddot{\epsilon}_{c}+K_{c c} \epsilon_{c}+\alpha_{c} \phi_{c}=f_{c 0}+f_{c 1} \sin (\omega t) \\
M_{b b} \ddot{\epsilon}_{b}+K_{b b} \epsilon_{b}+\alpha_{b} \phi_{b}=f_{b 0}+f_{b 1} \sin (\omega t) \\
m_{\varphi_{c}} \ddot{\phi}_{c}+k_{1 c} \phi_{c}+k_{2_{c c c}} \phi_{c}^{2}+k_{2_{b b c}} \phi_{b}^{2}+k_{3_{c c c c}} \phi_{c}^{3}+k_{3_{b b c c}} \phi_{b}^{2} \phi_{c}+\alpha_{c} \epsilon_{c}-\epsilon_{c} \mathcal{H}_{c}\left(\dot{\phi_{c}}, \dot{\phi_{b}}\right)=0 \\
m_{\varphi_{b}} \ddot{\phi}_{b}+k_{1 b} \phi_{b}+k_{2_{b c b}} \phi_{b} \phi_{c}+k_{3_{b c c b}} \phi_{b} \phi_{c}^{2}+k_{3_{b b b b}} \phi_{b}^{3}+\alpha_{b} \epsilon_{b}-\epsilon_{b} \mathcal{H}_{b}\left(\dot{\phi_{c}}, \dot{\phi_{b}}\right)=0
\end{array}\right.
$$

in which we set

$$
\begin{gathered}
M_{c c}=\int_{V} \rho\left[v^{2}\left(x_{1}^{2}+x_{2}^{2}\right)+x_{3}^{2}\right] \mathrm{d} V, \quad M_{b b}=\int_{V} \rho\left[v^{2}\left(x_{1}^{2}+x_{2}^{2}\right)+x_{3}^{2}\right]\left(\frac{x_{1}}{R}\right)^{2} \mathrm{~d} V \\
m_{\phi_{c}}=\int_{V} \rho_{\phi} \mathrm{d} V, \quad m_{\phi_{b}}=\int_{V} \rho_{\phi}\left(\frac{x_{1}}{R}\right)^{2} \mathrm{~d} V \\
K_{c c}=\int_{V} Y \mathrm{~d} V, \quad K_{b b}=\int_{V} Y\left(\frac{x_{1}}{R}\right)^{2} \mathrm{~d} V \\
k_{1 c}=\int_{V} k_{1} \mathrm{~d} V, \quad k_{1 b}=\int_{V} k_{1}\left(\frac{x_{1}}{R}\right)^{2} \mathrm{~d} V \\
k_{2_{c c c}}=\int_{V} k_{2} \mathrm{~d} V, \quad k_{2_{b b c}}=\int_{V} k_{2}\left(\frac{x_{1}}{R}\right)^{2} \mathrm{~d} V, \quad k_{2_{b c b}}=\int_{V} 2\left(\frac{x_{1}}{R}\right)^{2} k_{2} \mathrm{~d} V \\
k_{3_{c c c c}}=\int_{V} k_{3} \mathrm{~d} V, \quad k_{3_{b b c c}}=k_{3_{b c c b}}=\int_{V} 3\left(\frac{x_{1}}{R}\right)^{2} k_{3} \mathrm{~d} V, \quad k_{3_{b b b b}}=\int_{V}\left(\frac{x_{1}}{R}\right)^{4} k_{3} \mathrm{~d} V
\end{gathered}
$$




$$
\begin{gathered}
\mathcal{H}_{c}=H \int_{-R}^{R} \zeta(1-2 v) \tanh \left[\eta\left(\dot{\phi}_{b} \frac{x_{1}}{R}+\dot{\phi}_{c}\right)\right] \sqrt{R^{2}-x_{1}^{2}} \mathrm{~d} x_{1} \\
\mathcal{H}_{b}=H \int_{-R}^{R} \zeta(1-2 v) \tanh \left[\eta\left(\dot{\phi}_{b} \frac{x_{1}}{R}+\dot{\phi}_{c}\right)\right]\left(\frac{x_{1}}{R}\right)^{2} \sqrt{R^{2}-x_{1}^{2}} \mathrm{~d} x_{1}
\end{gathered}
$$

$Y=\frac{\mu(3 \lambda+2 \mu)}{\lambda+\mu}$ being the Young modulus and $\rho$ and $\rho_{\phi}$ being respectively the mass density of bulk material and an 'apparent' mass density linked to the kinetic energy related to the microstructural variable $\phi$. Moreover

$$
\alpha_{c}=\int_{V}[\sqrt{3} / 3(1+v) \alpha] \mathrm{d} V, \quad \alpha_{b}=\int_{V}[\sqrt{3} / 3(1+v) \alpha]\left(\frac{x_{1}}{R}\right)^{2} \mathrm{~d} V
$$

Finally, the uniform force that produces a pure compression, expressed by the terms $f_{c 0}$ and $f_{c 1}$ and the linear force, which produces a pure bending, expressed by $f_{b 0}$ and $f_{b 1}$, are given by $f_{c 0}=F_{c 0} \int_{S} H \mathrm{~d} S, f_{c 1}=F_{c 1} \int_{S} H \mathrm{~d} S$ and $f_{b 0}=F_{b 0} \int_{S} \frac{\left(x_{1}\right)^{2}}{R} H \mathrm{~d} S, f_{b 1}=F_{b 1} \int_{S} \frac{\left(x_{1}\right)^{2}}{R} H \mathrm{~d} S$.

The previous reduction procedure also entails other terms, which, however, are vanishing for simple symmetry arguments. For example, a term of the form

$$
M_{c b}=M_{b c}=\int_{V} \rho\left[v^{2}\left(x_{1}^{2}+x_{2}^{2}\right)+x_{3}^{2}\right] \frac{x_{1}}{R} \mathrm{~d} V=0,
$$

which derives from the substitution, vanishes as $x_{1}$ is integrated over a domain that is symmetrical with respect to the origin. In similar fashion, one can observe that a term $K_{b c}=K_{c b}$, and many others concerning the microstructure, also vanish.

Some more words have to be devoted to the form of the volumetric friction force, $\tau=$ $\zeta \operatorname{tr}(\boldsymbol{E}) \tanh (\eta \dot{\phi})$. In it, indeed, the trace of $\boldsymbol{E}$ appears, and it can be thought, in the small deformations approximation, as proportional to the compression between two sliding faces in a microcrack. Considering a pure compression, it is reasonable to assume that the dissipative internal friction force is suitably described by a term of this kind. In case of a pure bending, however, one can observe that, because the trace is positive in one of the two symmetric subsets in which the domain can be divided, such a form would imply the presence of a (locally) negative dissipation. In our physical interpretation, instead, a local dilatation would just entail that the dissipated energy vanishes, as there would be no friction between the faces of the microcracks. In the present work, the problem is simply solved by taking, in the considered eccentric compression, the bending coefficient $\epsilon_{b}$ as suitably smaller than the compression coefficient $\epsilon_{c}$, so as to have negative values everywhere for $\operatorname{tr}(\boldsymbol{E})$. In these hypotheses, which are of course very reasonable from an experimental point of view, the previously given expression for the internal friction stress always holds.

The ODEs system (4) obtained through the reducing procedure allows us to have a qualitative understanding of the dissipative behaviour of the considered model. In this regard, it should be noted, first of all, the presence of the coupling terms $k_{3 b b c c} \phi_{b}^{2} \phi_{c}$ and $k_{3 b c c b} \phi_{b} \phi_{c}^{2}$, respectively in the third and fourth equation, which play a relevant role in the interaction between compression and bending with regard to the microstructure. It is also evident the linearity of the dissipation terms with respect to $\epsilon_{c}$ and $\epsilon_{b}$, which ultimately derives from the term $\alpha \phi \sqrt{I_{2}^{(d)}}$ in the model (1).

The functions $\mathcal{H}_{c}\left(\dot{\phi}_{c}, \dot{\phi}_{b}\right)$ and $\mathcal{H}_{b}\left(\dot{\phi}_{c}, \dot{\phi}_{b}\right)$, defined in Equations (1) and (2), are plotted in Figures 1 and 2 considering both the sign (left) and hyperbolic tangent (right) for the description of friction. In the first case, because the integrands depend on $x_{1}$ only through the sign function, $\mathcal{H}_{c}$ and $\mathcal{H}_{b}$ have a constant value for those points $\left(\dot{\phi}_{c}, \dot{\phi}_{b}\right)$ for which $\left(\frac{x_{1}}{R} \dot{\phi}_{b}+\dot{\phi}_{c}\right)$ does not change sign when $x_{1}$ runs over $[-R, R]$, that is, in the triangular planar region that lies (clockwise) between the lines $l_{1}: \dot{\phi}_{c}=+\dot{\phi}_{b}$ and $l_{2}: \dot{\phi}_{c}=-\dot{\phi}_{b}$ - it can be noted that these lines do not depend on $R$ nor on $x_{1}$. The constant value is positive (negative) when $\dot{\phi}_{c}<0(>0)$. Because the integrands in Equations (10) and (11) are not continuous when replacing the hyperbolic tangent with the sign function, $\mathcal{H}_{c}$ and $\mathcal{H}_{b}$ are of course not differentiable (though continuous) along $l_{1,2}$, while using 

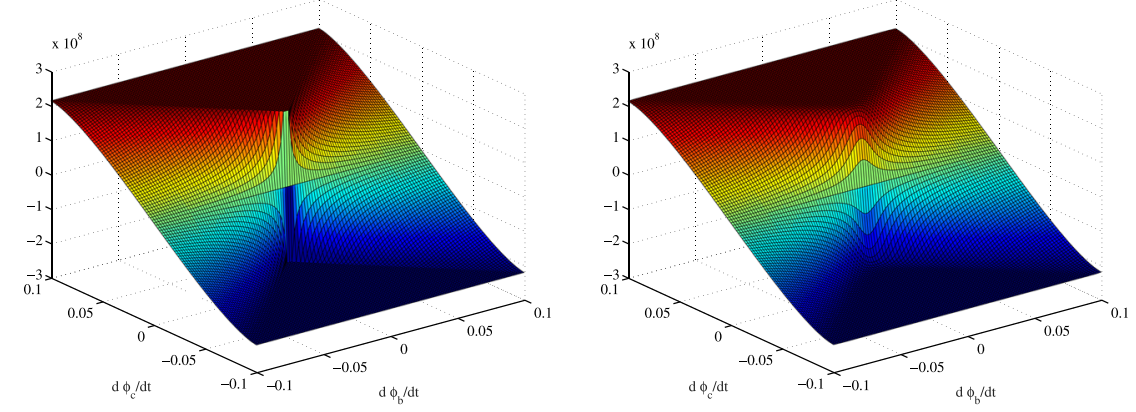

Figure 1. Functions $\mathcal{H}_{c}\left(\dot{\phi}_{c}, \dot{\phi}_{b}\right)$ considering the sign (left) and the tanh (right) functions.
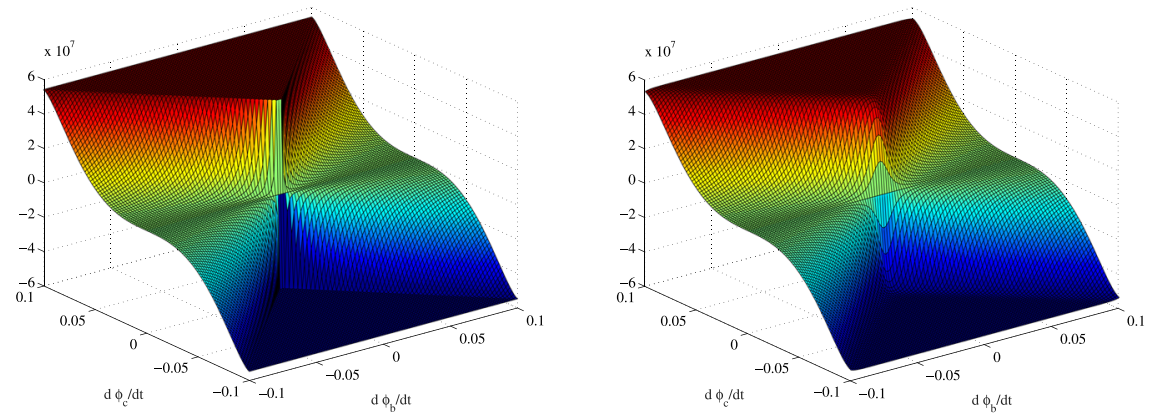

Figure 2. Functions $\mathcal{H}_{b}\left(\dot{\phi}_{c}, \dot{\phi}_{b}\right)$ considering the sign (left) and the tanh (right) functions.

the very form given in Equations (10) and (11), differentiability is recovered. Finally, note that because of the presence of the term $\left(\frac{x_{1}}{R}\right)^{2}, \mathcal{H}_{b}$ presents, with respect to $\mathcal{H}_{c}$, a more slowly increasing behaviour along the level lines $\dot{\phi}_{b}=$ const in the neighbourhood of $\dot{\phi}_{c}=0$. These mathematical considerations allow us to underline the physical meaning of the viscous-like behaviour connected with the use of the hyperbolic tangent, which as seen makes smoother, and thus physically more plausible, the description of the dissipation in our model, but does not change the general shape of the dissipation in the $\left(\dot{\phi}_{c}, \dot{\phi}_{b}\right)$ plane.

To conclude this section, we want to point out that our aim, in performing the reduction, was mainly to provide the previous qualitative analysis, while all numerical simulations considered in the next section will be computed considering the 3D model (1).

\section{NUMERICAL SIMULATIONS}

In this section, some numerical results concerning the model (1) in the case of pure compression and combined compression and bending of the type considered earlier are presented. The considered cylindric domain has a circular cross section of diameter $d=11.28 \mathrm{~cm}$ and a length $L=22 \mathrm{~cm}$. All the numerical simulations were performed using the commercial software COMSOL MULTIPHYSICS ${ }^{\circledR}$. We used an FEM method with a mesh with a maximum element size of $0.022 \mathrm{~m}$ and a time step of $0.005 \mathrm{~s}$.

In the first group of simulations, the case of pure compression is considered. The employed force has a sinusoidal compression component of amplitude $\approx 19 \mathrm{MPa}$, while a pre-stress of $1 \mathrm{MPa}$ was applied to ensure the contact action between the opposite faces of the microcracks (see the experimental section 4 for more on this). Our aim is to numerically evaluate how the parameter $\eta$ influences the cycle as well as the general behaviour of friction forces, $\epsilon$ and $\phi$. Concerning friction, the basic idea is to compare single cycles with different values for $\eta$. In this regard, let us start by observing that a natural condition to ask for when searching for a significant comparison is that cycles dissipate the same amount of energy. This implies that, once $\eta$ is chosen, the value of $\zeta$ is 
determined. Another specification is needed about the concept itself of 'cycle'. As we are in a dissipative regime, our physical variables are of course not exactly a periodic function of time. As it is well-known, anyway, one can prove that there is an asymptotic convergence toward a periodic regime [75]. In our simulations, we were indeed interested in these 'limit' cycles, which are independent of effects because of the initial conditions. Because the system converges towards a limit cycle in which the 'macro' elastic energy $\int_{t_{i}}^{t_{e}} \sigma_{i j} \dot{\epsilon}_{i j} \mathrm{~d} t$ vanishes, this same variable can reasonably be taken as a 'distance' from the asymptotic limit. In the simulations, it is actually observed that already in the second cycle the value of the 'macro' elastic energy is very close to zero, while from that value on, differences are within our numerical margin of error.

Concerning the physical meaning, a change in $\eta$ affects of course the amplitude of the interval of velocities for which the difference with the Coulomb-type case is significant. In Figure 3, the internal frictional force versus time is plotted, and three values for $\eta\left(2 \times 10^{2}, 2 \times 10^{4}\right.$ and $\left.8 \times 10^{4}\right)$ are considered. The chosen value for the dissipated energy is $0.687 \mathrm{~J}$. In the first case, the involved velocities are included in the aforementioned interval, and thus the simulation shows a viscous-type friction. In the third (and to a slightly lesser extent the second), the Coulomb-type friction regime is reached, as is observable from the marked slope of the decreasing part of the graph. Note that in the increasing part of the same graph a less sharp behaviour is observed, which is of course because of the dependence on $\operatorname{tr}(\boldsymbol{E})$.

In Figure 4, we studied the shape of cycles (in stress-displacement plotting) for the three already considered values for $\eta$ and the same fixed dissipated energy. As one can see, the effect of the increase in $\eta$ is able to affect the shape of the cycle, determining a widening in the top part of the graph while leaving more or less unchanged the bottom part.

Finally, variable $\phi$, which represents the relative displacement of two opposite faces of a microcrack, is plotted versus time in Figure 5. As one can observe, the relative displacement is of the same order of magnitude of the reported length of the microcracks [1], which is reasonable because in our assumptions in model (1) about the potential related to the microstructure, a cubic and quartic term accounting for big deformations are considered. As one can observe, differences in the values of $\eta$

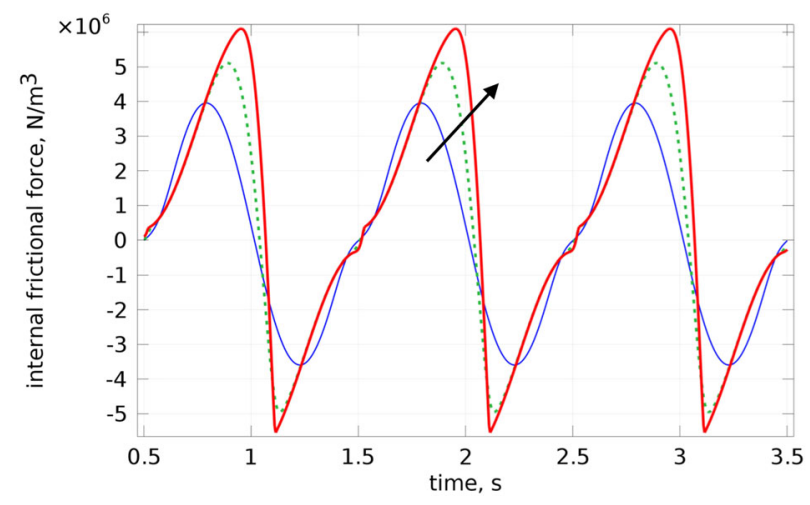

Figure 3. Time history of the volumetric frictional force. The arrow indicates higher values for $\eta\left(\eta=2 \times 10^{2}\right.$, $2 \times 10^{4}$ and $8 \times 10^{4}$ ).
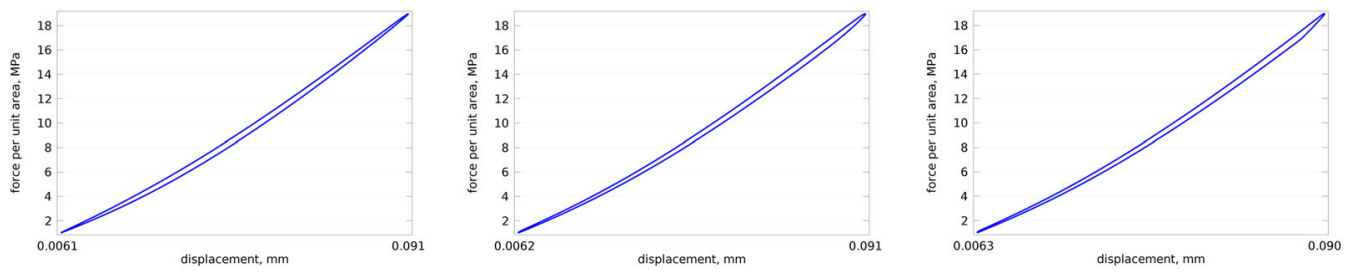

Figure 4. Influence of the parameter $\eta$ on the shape of cycles for $\eta=2 \times 10^{2}$, (left) $\eta=2 \times 10^{4}$, (middle) and $\eta=8 \times 10^{4}$ (right). 


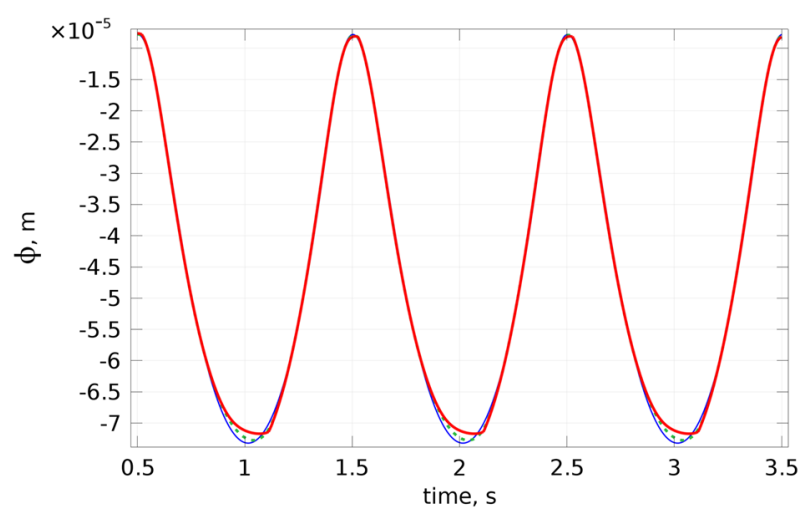

Figure 5. Time history of the relative microcrack displacement, $\phi$. As $\eta$ increases, $\phi$ gets sharper at the velocity reversal times, which in turn move forward. The effect is more evident in the minima.

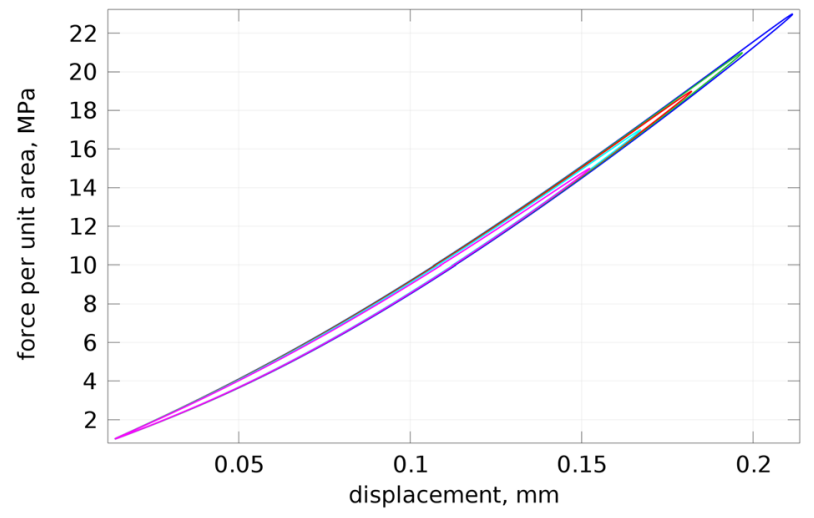

Figure 6. A 'regime' cycle in combined compressive and bending stress case. The different curves are relative to different points of the upper surface of the sample cylinder.

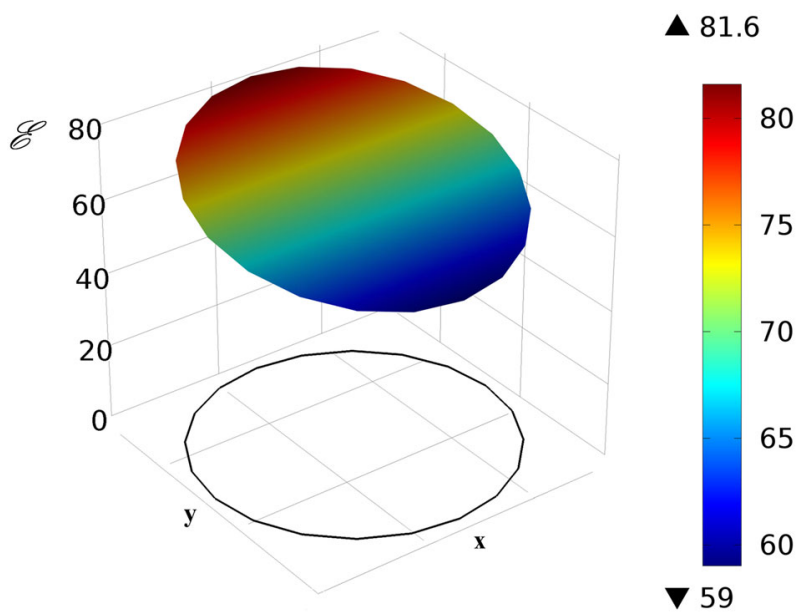

Figure 7. The distribution of the dissipated energy density $\left(\mathscr{E},\left[\mathrm{J} / \mathrm{m}^{2}\right]\right)$ as a function of the spatial coordinates for the upper surface of the cylinder.

only slightly affect $\phi$, which implies that the replacement of the sign function with the hyperbolic tangent does not affect the behaviour of the considered microstructure but just changes the way in which it influences friction, as one could reasonably ask for. 
In the last group of simulations, bending of the type used to derive the reduced form (4) is also considered. To the previously introduced compressive force, it was added a sinusoidal bending force of amplitude $2 \mathrm{MPa}$. In Figure 6, a 'regime' cycle is shown (in force density vs displacement plotting). Different curves are relative to different points of the upper surface of the cylinder (starting from the 'longest' cycle, $x_{1}$ is respectively equal to $R, \frac{R}{2}, 0,-\frac{R}{2}$ and $-R$ ). As one can see, there is no dramatic change in the general shape of the dissipation cycle; the main difference with the pure compression case being simply because of the fact that stress and strain are not uniform. The total dissipated energy in this case is $0.698 \mathrm{~J}$. This result shows that the difference between the two cases is actually negligible.

Finally, in Figure 7, the dissipated energy density for the upper surface of the cylinder is plotted. Specifically, the plotted variable is $\mathscr{E}=\int_{t_{0}}^{t_{0}+T} \sigma_{\text {ext }}(t) \cdot \dot{u}(H) \mathrm{d} t$, where $T$ is the cycle duration and $\sigma_{\mathrm{ext}}$ is the external applied force per unit surface area. The linearity in $x_{1}$ assumed for the microstructural variable $\phi$ is of course preserved, which gives us a geometrically evident argument in support of the previously mentioned negligibility of differences in the total dissipated energy.

Obviously, the considered numerical simulations can be very costly from a computational point of view, and even the formulation of the finite element problem can be non-trivial. For a set of numerical tools useful in this kind of context, the reader can refer to [76-80]. Moreover, the nonlinearities involved in Coulomb-type friction can easily entail instabilities, which can be treated with methods well-established in literature (see e.g. [81-87]).

\section{EXPERIMENTAL RESULTS}

In the present section, some basic experimental results supporting our model and our numerical simulations are provided.

The specimen is a simple cylinder having a ratio of length to diameter, $L / d$, equal to 2 . Considering both the desirability of small $L / d$ to avoid buckling and large $L / d$ to avoid end effects, the employed value represents a reasonable compromise for brittle materials, in which end effects are small [88]. The test specimen, as that considered in numerical simulations, has a circular cross section of diameter $d=11.28 \mathrm{~cm}$ and a length $L=22 \mathrm{~cm}$; its ends are provided with suitable capping to ensure that the test cylinder has a smooth, parallel and uniform bearing surfaces perpendicular to the applied axial load during compressive test.

In this regard, some words should be devoted to the possible influence of the capping/loading apparatus on the measured dissipated energy. A very sensible question, indeed, concerns the possibil-ity that part of the measured dissipation comes from friction occurring at the interface between the sample and the loading device. In our experiments, the sensors measuring $\epsilon$ (strain gauges) are posi-tioned at the center of the sample, far from the boundary effects, while the force applied in the region equipped with strain gauges is evidently independent from any possible dissipation occurring else-where. Therefore, even if a dissipation between the sample and the capping occurs (which is very possible), it would be not appreciated by the experimental apparatus. This point is confirmed con-sidering previous experiments performed without capping in which the measured dissipated energy was comparable [27]. Had some 'external' dissipation been measured in both cases, it should have affected, in principle, in different ways the measured total dissipation in the two cases, because there would have been no reason to assume that the two friction coefficients involved are equal. For these reasons, a direct measurement of the friction at the interface between the sample and the capping is not relevant to our aims.

The experimental results refer to two different recipes for concrete, that is, a standard concrete and a concrete modified by replacing a certain amount of cement (3\%) with rhombohedrical microparticles with specific weight of $2.71 \mathrm{~kg} / \mathrm{dm}^{3}$. In Tables I and II, the recipes for (respectively) standard and modified concrete are provided. Dissipation data (Table IV) refer to an average over six samples.

Experiments are performed under cyclic uniaxial (parallel to the axis of the specimen) compressive load. The test machine was controlled to impose a sinusoidal stress with a fixed frequency of $f=1 \mathrm{~Hz}$ for both tests. This low frequency is chosen to avoid inertial effects. More particularly, the considered load is 
Table I. The employed standard concrete.

\begin{tabular}{lcc}
\hline Composition & Weight ratio, kg & Ratio \\
\hline Sand & 21.25 & 35.1 \\
Gravel & 27.05 & 46.2 \\
Cement & 7.15 & 12.6 \\
Water & 4.8 & 6.14 \\
Total & 35.98 & 100 \\
\hline
\end{tabular}

Table II. The employed enriched concrete $(3 \%$ of added filler).

\begin{tabular}{lcc}
\hline Composition & Weight ratio, kg & Ratio \\
\hline Sand & 21.25 & 35.1 \\
Gravel & 27.05 & 46.2 \\
Cement & 6.94 & 11.51 \\
Water & 4.8 & 6.14 \\
Filler & 0.2145 & 0.356 \\
Total & 60.25 & 100 \\
\hline
\end{tabular}

$$
\sigma(t)=\left(\frac{\sigma_{\min }+\sigma_{\max }}{2}\right)+\left(\frac{\sigma_{\min }-\sigma_{\max }}{2}\right) \cos (2 \pi f t)
$$

where the values $\sigma_{\min }, \sigma_{\max }$ are related to the minimum and maximum forces applied, our force ranging from 19.62 to $176.58 \mathrm{kN}$. The peak load was chosen so as to avoid reaching a level of stress too close to the compressive strength of the material. On the other hand, the minimum level of compressive stress is large enough to ensure the interaction of opposite faces of the microcracks, on which the dissipated energy, in our physical interpretation, depends. Similarly to the previously considered numerical simulations, also for these experimental tests, the considered values are relative to cycles close enough to the asymptotic 'limit' cycle to avoid undesirable dependence on the initial conditions.

Our idea was to compare the dissipation and other physically relevant parameters between the two kinds of concrete to obtain a validation of our model as well as a proof of the meaningfulness of our physical interpretation of the dissipation. We, thus, considered a standard concrete and a concrete enriched by the addition of micro-filler. Of course, the addition of a filler can have different motivation and effects (see e.g. [89]). Roughly speaking, our reasoning is the following: one can reasonably expect that, by adding micro-particles of suitable characteristics and size, a given average relative displacement between the faces of a microcrack leads to an enhanced dissipation because of the extra friction due to the contact action between the faces themselves and the micro-particles. It can be remarked, moreover, that this kind of modification of concrete by means of suitable inert admixtures proposed in [27] are indeed founded on some precise theoretical considerations [62], which need to be further developed. Going in a little more detail, the idea can be described as follows: the concrete recipe is enriched in order to increase the dissipation capability without favouring the onset of plastic deformation or crack formation and growth. To fully develop this idea, an important theoretical effort is required: one can usefully refer to the methods used in the theory of structural modification (see e.g. [90-92] and references therein) or in the design of smart materials and structures (see e.g. [93-95] and references therein).

As the data reported in Tables III and IV show, in our case, the expected results are indeed observed, as both friction coefficients and the dissipated energy per cycle significantly increase in case of enriched concrete, and the results of the numerical simulations fit reasonably well with the provided experimental data. To make visually apparent the increase of the dissipated energy per cycle as a consequence of the addition of a micro-filler, a comparison between the cycles 
Table III. Material parameters of standard and enriched concrete.

\begin{tabular}{lccc}
\hline Parameters & Standard concrete & Enriched concrete & Unit \\
\hline$Y$ & $4.06 \times 10^{10}$ & $3.82 \times 10^{10}$ & $\mathrm{~N} / \mathrm{m}^{2}$ \\
$k_{1}$ & $1.86 \times 10^{12}$ & $1.86 \times 10^{12}$ & $\mathrm{~N} / \mathrm{m}^{4}$ \\
$k_{2}$ & $9.41 \times 10^{13}$ & $9.41 \times 10^{13}$ & $\mathrm{~N} / \mathrm{m}^{5}$ \\
$k_{3}$ & $1.22 \times 10^{20}$ & $9.01 \times 10^{19}$ & $\mathrm{~N} / \mathrm{m}^{6}$ \\
$\alpha$ & $2.89 \times 10^{11}$ & $2.87 \times 10^{11}$ & $\mathrm{~N} / \mathrm{m}^{3}$ \\
$\zeta$ & $5.50 \times 10^{11}$ & $4.17 \times 10^{11}$ & $\mathrm{~N} / \mathrm{m}^{3}$ \\
$\eta$ & $2.00 \times 10^{2}$ & $2.00 \times 10^{2}$ & $\mathrm{~s} / \mathrm{m}$ \\
\hline
\end{tabular}

Table IV. Dissipated energy for standard and enriched concrete.

\begin{tabular}{lc}
\hline Type & Dissipated energy per cycle \\
\hline Standard & $0.891 \pm 0.067 \mathrm{~J}$ \\
Enriched & $1.31 \pm 0.098 \mathrm{~J}$ \\
\hline
\end{tabular}
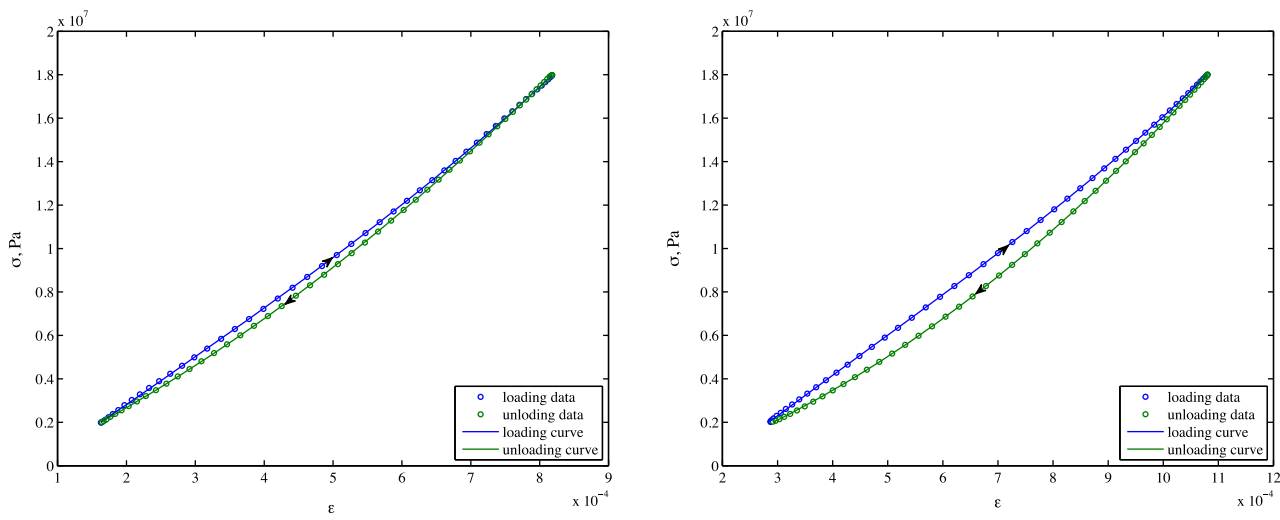

Figure 8. Dissipation loop for standard concrete (left) and for enriched concrete (right).

(in stress vs strain plotting) for standard and enriched concrete is provided in Figure 8. Of note, in Figure 8, measured data are represented by circlets while the loading and unloading curves are obtained by two second-order polynomial interpolations.

On the whole, both our theoretical model (1) and our physical interpretation, which links the dissipation to the relative sliding of the faces of the microcracks, as well as the meaningfulness of our numerical analysis, are fully justified by these experimental data.

\section{CONCLUSIONS}

The results presented in this work allow us to state that this (relatively) new line of investigation about concrete entails rich prospects for the development of a new understanding of dissipation phenomena and for useful - and not too remote - applications to Civil Engineering. The combination of a reasonably solid theoretical model with convincing numerical and experimental results represents, indeed, the better possible guarantee for the soundness and the future ambitions of a given research line. The results here presented represent of course a first theoretical step in the given direction, and further deepening, starting by possible refinements of the form of the Lagrangian, will have to be considered.

The case of combined compression and bending considered in the paper extended the simple compression case considered in [61], and the provided reduced model allowed us to qualitatively analyse the behaviour of the system. The numerical simulations showed the soundness and 
plausibility of our model, as the general behaviour of the considered variables was theoretically and numerically predictable and explainable, being moreover in excellent agreement with the qualitative considerations provided in the comments to the reduced model. In this connection, particularly interesting from a practical point of view was the highlighted negligibility of the difference with the case of pure compression with respect to the total dissipated energy per cycle.

The experimental results herein shown were intended as basic evidence in support of the proposed model and a further simple proof of the meaningfulness of our physical explanation of the dissipation, because the theoretical previsions concerning a comparison between a standard and an enriched concrete of the type considered were soundly confirmed.

As for possible future lines of investigation, a special attention will have to be devoted, in our opinion, to developments of the present model concerning a more accurate description of the dissipation in extreme regimes. As it is well-known, the dissipation, unlike what happens in our assumptions, is in general not exactly linear with the deformation but rather exhibits a threshold value within which it is not observed. Our physical interpretation of the dissipation, relying on the frictional sliding of the faces of the microcracks, is well-supported by this fact, because one can reasonably assume that, below certain levels of deformation, a non-negligible fraction of microcracks is not compressed enough to allow contact between the opposite faces. For this very reason, the applied cyclic force in the previously considered experimental tests had, as observed, a suitably large minimum level. An extension of the model, in order to accurately describe even a very small deformation regime, is of course of interest for the next future.

Moreover, a more detailed description of the character of the dissipation itself, capable to account for some rather non-standard effects (like a friction depending on the frequency of the external applied force) represents in our opinion a key step towards a successful application of the presented results to the real world.

\section{REFERENCES}

1. Mehta PK, Monteiro PJM. Concrete: Microstructure, Properties, and Materials, vol. 3. McGraw-Hill: New York, 2006.

2. Kotronis P, Collin F, Bésuelle P, Chambon R, Mazars J. Local second gradient models and damage mechanics: 1D post-localization studies in concrete specimens. In Bifurcations, Instabilities, Degradation in Geomechanics, Exadaktylos GE, Vardoulakis IG (eds). Springer: Berlin Heidelberg, 2007; 127-142.

3. Matsushima T, Chambon R, Caillerie D. Second gradient models as a particular case of microstructured models: a large strain finite elements analysis. Comptes Rendus de l'Academie de Sciences - Serie IIB: Mecanique, Physique, Chimie, Astronomie 2000; 328(2):179-186.

4. Quiligotti S, Maugin GA, dell'Isola F. Wave motions in unbounded poroelastic solids infused with compressible fluids. Zeitschrift für angewandte Mathematik und Physik ZAMP 2002; 53(6):1110-1138.

5. Quiligotti S, Maugin GA, dell'Isola F. An Eshelbian approach to the nonlinear mechanics of constrained solid-fluid mixtures. Acta Mechanica 2003; 160(1-2):45-60.

6. Pijaudier-Cabot G, Haidar K, Dubé J-F. Non-local damage model with evolving internal length. International Journal for Numerical and Analytical Methods in Geomechanics 2004; 28(7-8):633-652.

7. Bažant ZP, Zi G. Microplane constitutive model for porous isotropic rocks. International Journal for Numerical and Analytical Methods in Geomechanics 2003; 27(1):25-47.

8. Bourgeois F, Burlion N, Shao J-F. Modelling of elastoplastic damage in concrete due to desiccation shrinkage. International Journal for Numerical and Analytical Methods in Geomechanics 2002; 26(8):759-774.

9. Boukria Z, Limam A. Experimental damage analysis of concrete structures using the vibration signature-part II: located damage (crack). International Journal of Mechanics 2012; 6(1):28-34.

10. Nguyen GD, Houlsby GT. A coupled damage-plasticity model for concrete based on thermodynamic principles: Part I: model formulation and parameter identification. International Journal for Numerical and Analytical Methods in Geomechanics 2008; 32(4):353-389.

11. Nguyen GD, Houlsby GT. A coupled damage-plasticity model for concrete based on thermodynamic principles: Part II: non-local regularization and numerical implementation. International Journal for Numerical and Analytical Methods in Geomechanics 2008; 32(4):391-413.

12. Pietraszkiewicz W, Eremeyev V, Konopińska V. Extended non-linear relations of elastic shells undergoing phase transitions. ZAMM-Journal of Applied Mathematics and Mechanics/Zeitschrift für Angewandte Mathematik und Mechanik 2007; 87(2):150-159.

13. Dormieux L, Kondo D, Ulm F-J. Strength Homogenization, in Microporomechanics. John Wiley \& Sons: Chichester, UK, 2006. 


\section{SCERRATO ET AL.}

14. Auffray N, Bouchet R, Brechet Y. Strain gradient elastic homogenization of bidimensional cellular media. International Journal of Solids and Structures 2010; 47(13):1698-1710.

15. Bhattacharjee SS, Leger P. Seismic cracking and energy dissipation in concrete gravity dams. Earthquake Engineering \& Structural Dynamics 1993; 22(11):991-1007.

16. Brailsford AD. The role of internal stresses in internal friction. Physics Letters 1964; 12(2):92-93.

17. El Shamy U, Denissen C. Microscale energy dissipation mechanisms in cyclically-loaded granular soils. Geotechnical and Geological Engineering 2012; 30(2):343-361.

18. Beyrouthy J. Réduction 3D-1D d'un modèle viscoélastique en grandes déformations. Comptes Rendus Mathematique 2007; 345(4):239-243.

19. Solaria G, Pagnini LC, Piccardo G. A numerical algorithm for the aerodynamic identification of structures. Journal of Wind Engineering and Industrial Aerodynamics 1997; 69:719-730.

20. Eringen AC. Microcontinuum Field Theories I: Foundations and Solids. Springer-Verlag: New York, 1999.

21. Forest S, Sievert R. Nonlinear microstrain theories. International Journal of Solids and Structures 2006; 43(24):7224-7245.

22. Green AE, Rivlin RS. Simple force and stress multipoles. Archive for Rational Mechanics and Analysis 1964; 16(5):325-353.

23. Green AE, Rivlin RS. Multipolar continuum mechanics. Archive for Rational Mechanics and Analysis 1964; 17(2):113-147.

24. Mindlin RD. Micro-structure in linear elasticity. Archive for Rational Mechanics and Analysis 1964; 16(1):51-78.

25. Mindlin RD, Eshel NN. On first strain-gradient theories in linear elasticity. International Journal of Solids and Structures $1968 ; \mathbf{4}(1): 109-124$.

26. Neff P, Lankeit J, Madeo A. On Grioli's minimum property and its relation to Cauchy's polar decomposition. International Journal of Engineering Science 2014; 80:209-217.

27. Madeo A. Effect of micro-particle additions on frictional energy dissipation and strength of concrete. Ph.D. Thesis, Virginia Polytechnic Institute and State University, 2006.

28. Bowland AG, Weyers RE, Charney FA, Dowling NE, Murray TM, Ramniceanu A. Effect of vibration amplitude on concrete with damping additives. ACI Materials Journal 2012; 109(3):371-378.

29. dell'Isola F, Rosa L, Woźniak C. Dynamics of solids with microperiodic nonconnected fluid inclusions. Archive of Applied Mechanics 1997; 67:215-228.

30. dell'Isola F, Rosa L, Woźniak C. A micro-structured continuum modelling compacting fluid-saturated grounds: the effects of pore-size scale parameter. Acta Mechanica 1998; 127(1-4):165-182.

31. Misra A, Singh V. Micromechanical model for viscoelastic materials undergoing damage. Continuum Mechanics and Thermodynamics 2013; 25(2-4):343-358.

32. Misra A, Yang Y. Micromechanical model for cohesive materials based upon pseudo-granular structure. International Journal of Solids and Structures 2010; 47(21):2970-2981.

33. dell'Isola F, Batra RC. Saint-Venant's problem for porous linear elastic materials. Journal of Elasticity 1997; 47(1):73-81.

34. Sciarra G, dell'Isola F, Hutter K. A solid-fluid mixture model allowing for solid dilatation under external pressure. Continuum Mechanics and Thermodynamics 2001; 13(5):287-306.

35. Alibert J-J, Seppecher P, dell'Isola F. Truss modular beams with deformation energy depending on higher displacement gradients. Mathematics and Mechanics of Solids 2003; 8(1):51-73.

36. Ferretti M, Madeo A, dell'Isola F, Boisse P. Modeling the onset of shear boundary layers in fibrous composite reinforcements by second-gradient theory. ZAMP-Zeitschrift für angewandte Mathematik und Physik 2014; $\mathbf{6 5}(3)$ : $587-612$.

37. Madeo A, dell'Isola F, Darve F. A continuum model for deformable, second gradient porous media partially saturated with compressible fluids. Journal of the Mechanics and Physics of Solids 2013; 61(11):2196-2211.

38. Nadler B, Papadopoulos P, Steigmann DJ. Multiscale constitutive modeling and numerical simulation of fabric material. International Journal of Solids and Structures 2006; 43(2):206-221.

39. Sciarra G, dell'Isola F, Coussy O. Second gradient poromechanics. International Journal of Solids and Structures 2007; 44(20):6607-6629.

40. Yang Y, Ching WY, Misra A. Higher-order continuum theory applied to fracture simulation of nanoscale intergranular glassy film. Journal of Nanomechanics and Micromechanics 2011; 1(2):60-71.

41. Yang Y, Misra A. Higher-order stress-strain theory for damage modeling implemented in an element-free Galerkin formulation. CMES-Computer Modeling in Engineering \& Sciences 2010; 64(1):1-36.

42. Batra RC, dell'Isola F, Ruta GC. Second-order solution of Saint-Venant's problem for an elastic bar predeformed in flexure. International Journal of Non-Linear Mechanics 2005; 40(2):411-422.

43. Neff P, Ghiba I-D, Madeo A, Placidi L, Rosi G. A unifying perspective: the relaxed linear micromorphic continuum. Continuum Mechanics and Thermodynamics 2013; 26(5):639-681.

44. Pietraszkiewicz W, Eremeyev VA. On vectorially parameterized natural strain measures of the non-linear Cosserat continuum. International Journal of Solids and Structures 2009; 46(11):2477-2480.

45. Madeo A, Placidi L, Rosi G. Towards the design of metamaterials with enhanced damage sensitivity: second gradient porous materials. Research in Nondestructive Evaluation 2014; 25(2):99-124.

46. Andreaus U, Giorgio I, Lekszycki T. A 2-D continuum model of a mixture of bone tissue and bio-resorbable material for simulating mass density redistribution under load slowly variable in time. ZAMM - Zeitschrift für Angewandte Mathematik und Mechanik/Journal of Applied Mathematics and Mechanics 2014; 94(12):978-1000. 
47. Lekszycki T, dell'Isola F. A mixture model with evolving mass densities for describing synthesis and resorption phenomena in bones reconstructed with bio-resorbable materials. ZAMM-Journal of Applied Mathematics and Mechanics/Zeitschrift für Angewandte Mathematik und Mechanik 2012; 92(6):426-444.

48. Savage JC, Byerlee JD, Lockner DA. Is internal friction friction? Geophysical Research Letters 1996; 23(5):487-490.

49. Spears CJ, Feltham P. On the amplitude-independent internal friction in crystalline solids. Journal of Materials Science 1972; 7(8):969-971.

50. Zener C. Internal friction in solids. Proceedings of the Physical Society 1940; 52(1):152-166.

51. Wegel RL, Walther H. Internal dissipation in solids for small cyclic strains. Journal of Applied Physics 1935; 6(4):141-157.

52. Kimball AL, Lovell DE. Internal friction in solids. Physical Review 1927; 30(6):948-959.

53. Fantozzi G, Ritchie IG. Internal friction caused by the intrinsic properties of dislocations. Le Journal de Physique Colloques 1981; 42(C5):C5-3.

54. Fantozzi G, Esnouf C, Benoit W, Ritchie IG. Internal friction and microdeformation due to the intrinsic properties of dislocations: the bordoni relaxation. Progress in Materials Science 1982; 27(3):311-451.

55. Yu C-T, Kobayashi AS, Hawkins NM. Energy-dissipation mechanisms associated with rapid fracture of concrete. Experimental Mechanics 1993; 33(3):205-211.

56. D'Annibale F, Luongo A. A damage constitutive model for sliding friction coupled to wear. Continuum Mechanics and Thermodynamics 2013; 25(2-4):503-522.

57. Rinaldi A, Placidi L. A microscale second gradient approximation of the damage parameter of quasi-brittle heterogeneous lattices. ZAMM-Journal of Applied Mathematics and Mechanics/Zeitschrift für Angewandte Mathematik und Mechanik 2013; 94(10):862-877.

58. Andreaus U, Baragatti P. Fatigue crack growth, free vibrations, and breathing crack detection of aluminium alloy and steel beams. The Journal of Strain Analysis for Engineering Design 2009; 44(7):595-608.

59. Hillerborg A, Modéer M, Petersson P-E. Analysis of crack formation and crack growth in concrete by means of fracture mechanics and finite elements. Cement and Concrete Research 1976; 6(6):773-781.

60. Contrafatto L, Cuomo M. A globally convergent numerical algorithm for damaging elasto-plasticity based on the multiplier method. International Journal for Numerical Methods in Engineering 2005; 63(8):1089-1125.

61. Scerrato D, Giorgio I, Madeo A, Limam A, Darve F. A simple non-linear model for internal friction in modified concrete. International Journal of Engineering Science 2014; 80:136-152.

62. Pensée V, Kondo D, Dormieux L. Micromechanical analysis of anisotropic damage in brittle materials. Journal of Engineering Mechanics 2002; 128(8):889-897.

63. Boma MB, Brocato M. A continuum model of micro-cracks in concrete. Continuum Mechanics and Thermodynamics 2010; 22(2):137-161.

64. Adelaide L, Richard B, Ragueneau F, Cremona C. Thermodynamical admissibility of a set of constitutive equations coupling elasticity, isotropic damage and internal sliding. Comptes Rendus Mécanique 2010; 338(3):158-163.

65. Federico S, Grillo A, Wittum G. Considerations on incompressibility in linear elasticity. Il Nuovo cimento della Società italiana di fisica. C 2009; 32(1):81-87.

66. Federico S, Grillo A, Imatani S. The linear elasticity tensor of incompressible materials. Mathematics and Mechanics of Solids 2014. DOI: 1081286514550576.

67. Altenbach H, Eremeyev VA, Morozov NF. Surface viscoelasticity and effective properties of thin-walled structures at the nanoscale. International Journal of Engineering Science 2012; 59:83-89.

68. De Simone A, Luongo A. Nonlinear viscoelastic analysis of a cylindrical balloon squeezed between two rigid moving plates. International Journal of Solids and Structures 2013; 50(14):2213-2223.

69. dell'Isola F, Hutter K. What are the dominant thermomechanical processes in the basal sediment layer of large ice sheets? Proceedings of the Royal Society of London. Series A: Mathematical, Physical and Engineering Sciences 1998; 454(1972):1169-1195.

70. Bastien J, Schatzman M, Lamarque C-H. Study of some rheological models with a finite number of degrees of freedom. European Journal of Mechanics-A/Solids 2000; 19(2):277-307.

71. Lamarque C-H, Bernardin F, Bastien J. Study of a rheological model with a friction term and a cubic term: deterministic and stochastic cases. European Journal of Mechanics-A/Solids 2005; 24(4):572-592.

72. Andreaus U, Ruta GC. A review of the problem of the shear centre (s). Continuum Mechanics and Thermodynamics 1998; 10(6):369-380.

73. Germain P. Sur l'application de la méthode des puissances virtuelles en mécanique des milieux continus. Comptes Rendus de l'Académie des Sciences (Paris) - Série A 1972; 274:1051-1055.

74. Germain P. The method of virtual power in continuum mechanics. Part 2: microstructure. SIAM Journal on Applied Mathematics 1973; 25(3):556-575.

75. Volpato M. Sull'esistenza di soluzioni periodiche per equazioni differenziali ordinarie del secondo ordine. Rendiconti del Seminario Matematico della Università di Padova 1956; 25:371-385.

76. Cazzani A, Malagù M, Turco E. Isogeometric analysis of plane-curved beams. Mathematics and Mechanics of Solids 2014. DOI: 1081286514531265.

77. Greco L, Cuomo M. An implicit G1 multi patch B-spline interpolation for Kirchhoff-Love space rod. Computer Methods in Applied Mechanics and Engineering 2014; 269:173-197.

78. Greco L, Cuomo M. B-spline interpolation of Kirchhoff-Love space rods. Computer Methods in Applied Mechanics and Engineering 2013; 256:251-269. 


\section{SCERRATO ET AL}

79. Greco L, Cuomo M. Consistent tangent operator for an exact Kirchhoff rod model. Continuum Mechanics and Thermodynamics 2014. DOI: 10.1007/s00161-014-0361-x.

80. Turco E, Caracciolo P. Elasto-plastic analysis of Kirchhoff plates by high simplicity finite elements. Computer Methods in Applied Mechanics and Engineering 2000; 190(5):691-706.

81. Belyakov AO, Seyranian AP, Luongo A. Dynamics of the pendulum with periodically varying length. Physica D: Nonlinear Phenomena 2009; 238(16):1589-1597.

82. Di Egidio A, Luongo A, Paolone A. Linear and non-linear interactions between static and dynamic bifurcations of damped planar beams. International Journal of Non-Linear Mechanics 2007; 42(1):88-98.

83. Luongo A, Zulli D, Piccardo G. On the effect of twist angle on nonlinear galloping of suspended cables. Computers \& Structures 2009; 87(15):1003-1014.

84. Luongo A. Mode localization in dynamics and buckling of linear imperfect continuous structures. Nonlinear Dynamics 2001; 25(1-3):133-156.

85. Eremeev VA, Freidin AB, Sharipova LL. Nonuniqueness and stability in problems of equilibrium of elastic two-phase bodies. Doklady Physics Springer, 2003; 48(7):359-363.

86. Jamal M, Lahlou L, Midani M, Zahrouni H, Limam A, Damil N, Potier-Ferry M. A semi-analytical buckling analysis of imperfect cylindrical shells under axial compression. International Journal of Solids and Structures 2003; 40(5):1311-1327.

87. Michel G, Limam A, Jullien JF. Buckling of cylindrical shells under static and dynamic shear loading. Engineering Structures 2000; 22(5):535-543.

88. Dowling NE. Mechanical Behavior of Materials. Pearson: Upper Saddle River, New Jersey, 2012.

89. Misra A, Biswas D, Upadhyaya S. Physico-mechanical behavior of self-cementing class C fly ash-clay mixtures. Fuel 2005; 84(11):1410-1422.

90. Carcaterra A, Akay A. Dissipation in a finite-size bath. Physical Review E 2011; 84(1):011121-011124.

91. Culla A, Sestieri A, Carcaterra A. Energy flow uncertainties in vibrating systems: definition of a statistical confidence factor. Mechanical Systems and Signal Processing 2003; 17(3):635-663.

92. Carcaterra A, Akay A. Theoretical foundations of apparent-damping phenomena and nearly irreversible energy exchange in linear conservative systems. The Journal of the Acoustical Society of America 2007; 121(4):1971-1982.

93. Vidoli S, dell'Isola F. Modal coupling in one-dimensional electromechanical structured continua. Acta Mechanica 2000; 141(1-2):37-50.

94. Vidoli S, dell'Isola F. Vibration control in plates by uniformly distributed PZT actuators interconnected via electric networks. European Journal of Mechanics-A/Solids 2001; 20(3):435-456.

95. Porfiri M, dell'Isola F, Santini E. Modeling and design of passive electric networks interconnecting piezoelectric transducers for distributed vibration control. International Journal of Applied Electromagnetics and Mechanics 2005; 21(2):69-87. 